\title{
Variabilidade espacial dos atributos químicos em terra preta de índio cultivada na região de Novo Aripuanã, AM
}

\section{Spatial variability of chemical atributes in cultivated archeological dark earth in Novo Aripuanã region}

\author{
Selma Ferreira Viana ${ }^{1}$; Milton César Costa Campos ${ }^{2}$; José Maurício da Cunha ${ }^{3}$; William Barros do Nascimento ${ }^{4}$ : Antonio \\ Francisco de Mendonça Júnior ${ }^{5}$
}

Resumo: Terras Pretas de Índio são solos de uma fertilidade notoriamente superior à grande maioria dos solos típicos da região Amazônica. Apresentam em sua composição fragmentos de cerâmica e artefatos indígenas incorporados a matriz dos horizontes superficiais, dispõem de alta estabilidade e persistência mantendo sua fertilidade mesmo depois de vários anos. Nesse sentido, o presente trabalho tem como objetivo avaliar a variabilidade espacial dos atributos químicos do solo em área de Terra Preta de Índio sob cultivo de feijão em Novo Aripuanã, AM. Foi realizado o mapeamento de uma malha de $20,0 \mathrm{x}$ 72,0 m, com espaçamento regular de 5,0 x 4,0 m, nas profundidades de amostragem 0,0 - 0,05, 0,05 - 0,10 e 0,10 - 0,20 m, totalizando 90 pontos. Foram realizadas análises químicas como: pH em água, fósforo (P), concentração de alumínio (Al), e acidez potencial $(\mathrm{H}+\mathrm{Al})$. Os dados foram analisados utilizando-se técnicas estatísticas descritivas e de geoestatística. Os químicos estudados apresentaram estrutura de dependência espacial, com exceção da variável alumínio que indicou efeito pepita puro. Os valores de alcance foram diferentes variando de 8,73 a 81,00 m, estando dentro do limite estabelecido pela malha de estudo.

Palavras-Chave: Atributos do solo. Geoestatística. Solos antrópicos.

Abstract: Archeological dark earth are soils of higher fertility than majority of typical soils from the Amazon region. These have in its composition, pottery fragments and indian artifacts embedded in the matrix of the surface horizons, present high stability and persistence, which maintains its fertility even after several years. The objective of the study was to evaluate the spatial variability of chemical atributes in archeological dark earth under bean cultivation, Novo Aripuanã, Amazonas. Was conducted mapping a mesh of $20.0 \times 72.0 \mathrm{~m}$, with regular spacing of $5.0 \times 4.0 \mathrm{~m}$, in the sampling depths from 0.0-0.05, 0.050.10 and $0.10-0.20 \mathrm{~m}$, totaling 90 points. Were performed some chemical analyzes: $\mathrm{pH}$, phosphorus (P), aluminum concentration $(\mathrm{Al})$, and potential acidity $(\mathrm{H}+\mathrm{Al})$. Data were analyzed using descriptive statistics techniques and geostatistical. The studied chemical had spatial dependence structure, except for the aluminum that indicated pure nugget effect. The range values were different ranging from 8.73 to $81.00 \mathrm{~m}$, and within the limits established by the mesh study.

Keywords: Anthropic soils. Geostatistics. Soil properties.

\footnotetext{
*Autor para correspondência

Recebido para publicação em 20/06/2016; aprovado em 07/08/2016

${ }^{1}$ Graduação em Agronomia. Universidade Federal do Amazonas, UFAM, Brasil. E-mail: selmaviana1993@hotmail.com

${ }^{2}$ Professor. Universidade Federal do Amazonas, UFAM, Brasil.

${ }^{3}$ Professor. Universidade Federal do Amazonas, UFAM, Brasil.

${ }^{4}$ Graduação em Engenharia Ambiental. Universidade Federal do Amazonas, UFAM, Brasil.

${ }^{5}$ Professor. Universidade Federal de Campina Grande, UFCG, Brasil.
} 


\section{INTRODUÇÃO}

As Terras Pretas de Índio (TPI's), também conhecidas como Terra Preta Arqueológica (TPA), ou simplesmente Terra Preta (TP) são solos de uma fertilidade notoriamente superior à grande maioria dos solos típicos da região Amazônica. Encontrados em diversas regiões da Amazônia, são solos que sofreram atividade humana pré-histórica intensa na forma de intensos acúmulos de resíduos que modificaram significativamente suas propriedades (FAO, 2011).

A Terra Preta é caracterizada por apresentar elevada fertilidade natural resultante de prolongada ocupação antrópica, sendo encontradas em meio à vasta extensão de terras, na grande maioria extremamente pobre em nutrientes (CORRÊA, 2007). As Terras Pretas de Índios (TPI's) são encontrados no Norte do Brasil, em manchas com um tamanho médio de 20 ha (GLASER, 2007). Ao contrário dos solos encontrados em regiões adjacentes, apresentando tipicamente baixa retenção de nutrientes devido às fortes chuvas, altas temperaturas e umidade, que geralmente aceleram o processo de degradação da matéria orgânica do solo (MOS), as TPI's apresentam alta estabilidade e persistência (GLASER, 2002; LIANG, et al, 2006; CHENG, 2009) mantendo sua fertilidade mesmo depois de vários anos.

A análise detalhada da variabilidade espacial dos atributos do solo pode indicar alternativas de manejo para a redução dos efeitos da sua variabilidade sobre a produção das culturas (WERNER et al., 2007). O conhecimento da variabilidade das propriedades do solo e das culturas, no espaço e no tempo, é considerado, o princípio básico para o

Figura 1- Mapa de localização do município de Novo Aripuanã - AM. manejo preciso das áreas agrícolas, qualquer que seja sua escala (GREGO; VIEIRA, 2005).

A análise geoestatística permite detectar a existência da variabilidade e distribuição espacial de variáveis do solo, constituindo assim uma importante ferramenta na análise e descrição detalhada dos atributos do solo (CAMPOS et al., 2007). Além disso, o estudo da dependência espacial apresenta-se como uma alternativa não só para reduzir os efeitos da variação do solo na produção das culturas, mas também, para estimar respostas das propriedades do solo em função de determinadas práticas de manejo, permitindo a redução dos efeitos da variabilidade horizontal e vertical do solo, em muitos casos, pode influenciar na interpretação de seus efeitos (SOUZA et al., 2004).

Dessa forma, o objetivo deste estudo foi avaliar a variabilidade espacial dos atributos químicos em Terra Preta de Índio sob cultivo de feijão guandu na região de Novo Aripuanã, Amazônia.

\section{MATERIAL E MÉTODOS}

O estudo foi realizado em uma área situada na região do município de Novo Aripuanã, sul do Estado do Amazonas, sob as coordenadas geográficas de $7^{\circ} 30^{\prime} 24^{\prime \prime} \mathrm{S}$ e $63^{\circ} 04^{\prime} 56^{\prime \prime}$ W. A zona climática da região, segundo a classificação de Köppen, pertence ao grupo A (Clima Tropical Chuvoso) e tipo climático AM (chuvas do tipo monção), apresentando um período seco de pequena duração, com precipitação média anual varia entre $2.500 \mathrm{~mm}$, e com período chuvoso iniciando em outubro e prolongando-se até junho. As médias anuais de temperatura variam em torno de $25^{\circ} \mathrm{C}$ e $27^{\circ} \mathrm{C}$, e a umidade relativa do ar varia entre 85 e $90 \%$.

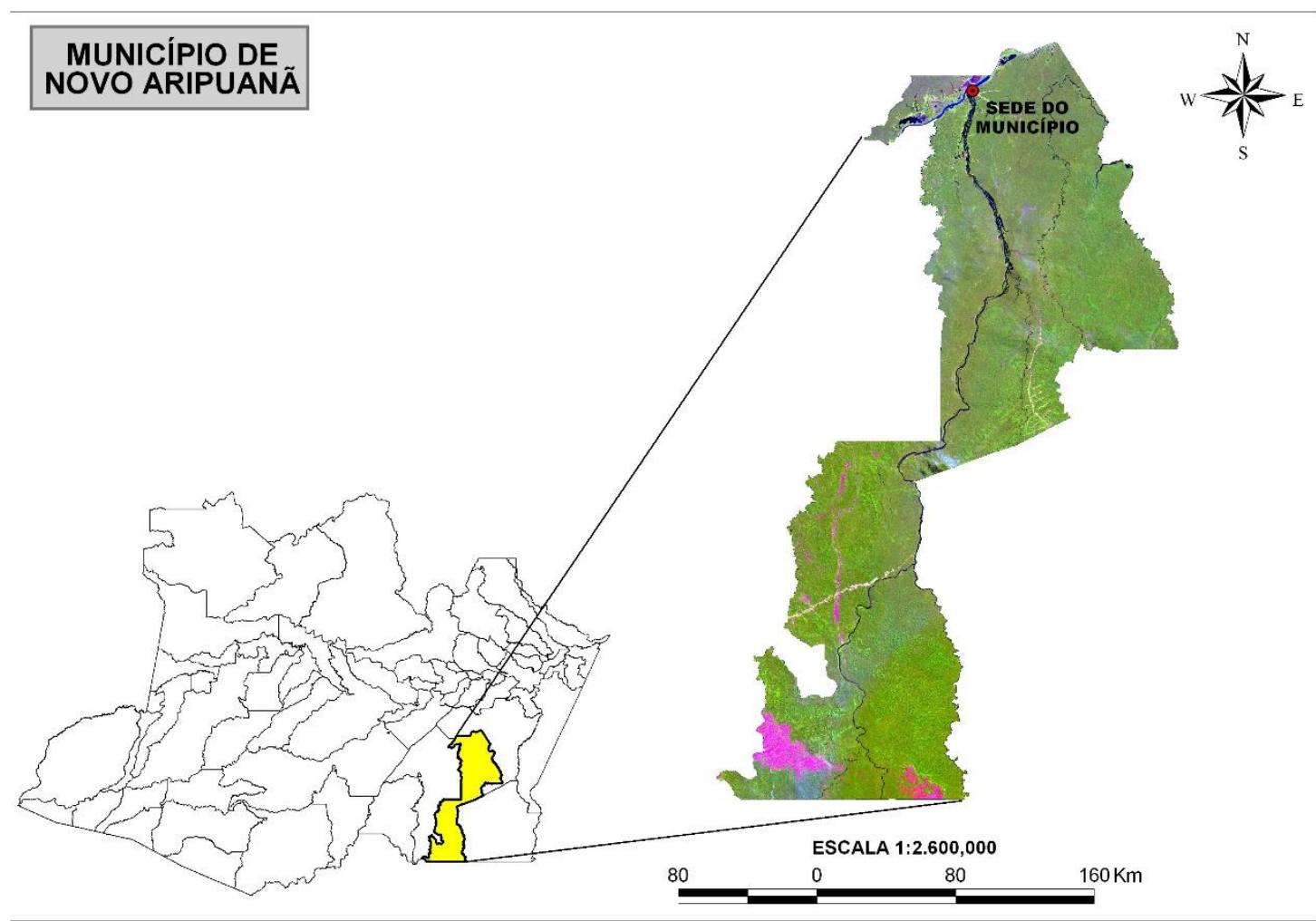

Fonte: Autor (2016). 
Segundo Lima et al. (2002) e Cunha et al. (2007), encontram-se na região áreas conhecidas com Terra Preta Arqueológicas relacionados com locais de antigos assentamentos, contendo artefatos culturais, e apresentam elevada fertilidade natural.

A vegetação característica desta região é a Floresta Tropical Densa constituída por árvores adensadas e multiestratificadas entre 20 a 50 metros de altura. De acordo com Campos (2012) as paisagens predominantes dessa região são os Campos Naturais, Campos Naturais/ Florestas e Florestas.

As amostras foram coletadas em uma área sob cultivo de feijão guandu, em uma malha de 20,0 x 72,0 m, com espaçamento regular de $5,0 \times 4,0 \mathrm{~m}$, nas profundidades de amostragem 0,0 - 0,05, 0,05 - 0,10 e 0,10 - 0,20 m, totalizando 90 pontos em cada uma das profundidades, e totalizando 270 pontos amostrais nas 3 profundidades.

$\mathrm{O} \mathrm{pH}$ foi determinado potenciometricamente utilizando-se água, em relações solo: solução 1:2,5. Alumínio trocáveis foi extraído por $\mathrm{KCl}$ (cloreto de potássio). O fósforo disponível, foi extraídos por Mehlich ${ }^{-1}$, a acidez potencial $(\mathrm{H}+\mathrm{Al})$ foi extraída com solução tamponada a pH 7,0 de acetato de cálcio utilizando-se metodologia proposta pela EMBRAPA (1997). Os atributos do solo foram analisados por meio da análise estatística descritiva, sendo determinados a média, mediana, o coeficiente de variação, o coeficiente de assimetria e curtose. As hipóteses de normalidade dos dados foram testadas pelo teste de Kolmogorov-Smirnov (KS) $(\mathrm{p} \leq 0,05)$, por meio do software computacional Minitab 14 (MINITAB, 2000).

A geoestatística foi usada para avaliar a variabilidade espacial dos atributos estudados, segundo VIEIRA et al. (1983). Para se fazer a análise geoestatística, foi necessário saber se há dependência espacial ou não dos atributos estudados, o que poderá ser verificado por meio do gráfico do semivariograma.

O semivariograma é uma representação gráfica entre a semivariância $\mathrm{y}(\mathrm{h})$, representada na coordenada $\mathrm{Y}$, em função de uma determinada distância (h), representada na coordenada $X$. Para a caracterização da variabilidade espacial, foi utilizada a análise geoestatística (ISAAKS; SRIVASTAVA, 1989). Com base na pressuposição de estacionariedade da hipótese intrínseca, a qual o semivariograma é estimada por:

$$
\hat{\gamma}(h)=\frac{1}{2 N(h)} \sum_{i=1}^{N(h)}\left[Z\left(x_{i}\right)-Z\left(x_{i}+h\right)\right]^{2}
$$

Sendo: $\gamma(\mathrm{h})$ - valor da semivariância para uma distância $\mathrm{h} ; \mathrm{N}(\mathrm{h})$ - número de pares envolvidos no cálculo da semivariância; $\mathrm{Z}\left(\mathrm{x}_{\mathrm{i}}\right)$ - valor do atributo $\mathrm{Z}$ na posição $\mathrm{x}_{\mathrm{i}}$; $\mathrm{Z}\left(\mathrm{x}_{\mathrm{i}}+\mathrm{h}\right)$ - valor do atributo $\mathrm{Z}$ separado por uma distância $\mathrm{h}$ da posição xi.

Os ajustes dos modelos experimentais ao semivariograma foram baseadas no maior valor do coeficiente de determinação e no menor valor da raiz quadrada do erro médio, e a escolha do melhor ajuste foi realizada utilizando a técnica conhecida por "jack-knifing" de acordo com Vieira et al. (2002).

Para analisar o grau da dependência espacial dos atributos do solo, foi utilizado a classificação de Cambardella et al., (1994), em que são considerados dependência espacial forte os semivariograma que têm um efeito pepita $<25 \%$ do patamar, moderada quando está entre 25 e $75 \%$ e fraca, $>75 \%$.

\section{RESULTADOS E DISCUSSÃO}

Os resultados referentes a análise descritiva para as variáveis $\mathrm{pH}$ em água, $\mathrm{H}+\mathrm{Al}, \mathrm{Al}^{3+}, \mathrm{P}$ disponível nas profundidades de $0,0-0,05 \mathrm{~m} ; 0,05-0,10 \mathrm{~m} ; 0,10-0,20 \mathrm{~m}$ são apresentados na tabela 1 . Observa-se, que os valores das medidas de tendência central (média, mediana) são semelhantes para todas as variáveis, o que prova que a distribuição é simétrica para esses atributos do solo, o que pode ser confirmado pelos valores de assimetria e curtose próximos de zero. A proximidade dos valores média e mediana assegura uma distribuição simétrica dos dados, isso significa que a dispersão dos valores não apresenta caudas muito alongadas, o que poderia comprometer a análise geoestatística, ocorre somente que os valores de assimetria e curtose são sensíveis a valores extremos de forma que um único valor pode exercer grande influência nos seus resultados (DIGGLE; RIBEIRO JÚNIOR, 2007). As medidas de dispersão, próximas de 0 e 3 para assimetria e curtose, respectivamente, mostram distribuição normal, conforme FARIAS (1999).

Com relação ao teste de normalidade dos dados submetidos ao teste de Kolmogorov-Smirnov (Tabela 1), o qual mede a distância máxima entre os resultados de uma distribuição a ser testada e os resultados associados à distribuição hipoteticamente verdadeira (GONÇALVES et al., 2001), verificou-se que todas as variáveis foram significativas ao nível de $5 \%$ de probabilidade, com exceção da variável fósforo na profundidade $0,05-0,10 \mathrm{~m}$. A normalidade dos dados não é uma exigência da geoestatística, entretanto é conveniente apenas que a distribuição não apresente caudas muito alongadas, o que poderia comprometer as estimativas da krigagem, as quais são baseadas nos valores médios (ISAAKS; SRIVASTAVA, 1989).

Pelos limites de coeficiente de variação (Tabela 1) propostos por Warricke Nielsen (1980) para a classificação de variáveis do solo (CV < $12 \%),(12 \%<\mathrm{CV}>60 \%)$ e $(\mathrm{CV}>$ $60 \%$ ) para baixa, média e alta variabilidade, respectivamente, diz-se que os atributos apresentaram baixa variabilidade para pH em $\mathrm{H}_{2} \mathrm{O}$, moderada variabilidade para acidez potencial, fósforo e alumínio, com exceção na profundidade 0,00-0,05 $\mathrm{m}$ da variável alumínio, apresentado alta variabilidade. Segundo Nogueira (2007), um coeficiente de variação maior que $35 \%$ revela que a série é heterogênea e a média tem pouco significado; se for maior que $65 \%$, a série é muito heterogênea e a média não tem significado algum, porém se for menor que $35 \%$ a série é homogênea e a média tem significado, podendo ser utilizada como representativa da série de onde foi obtida. Desta forma, pode-se dizer que a $\mathrm{pH}$ em $\mathrm{H}_{2} \mathrm{O}$ apresentou série de dados homogêneos e a média apresentando significado, contradizendo as demais variáveis que apresentaram série de dados heterogêneas. 
Tabela 1. Estatística descritiva de $\mathrm{pH}$ em água, $\mathrm{H}+\mathrm{Al}, \mathrm{Al}^{3+}, \mathrm{P}$ disponivel do solo em área de Terra Preta Arqueológica sob cultivo de feijão no município de Novo Aripuanã - AM.

\begin{tabular}{|c|c|c|c|c|}
\hline Estatística Descritiva & $\mathrm{pH}$ em $\mathrm{H}_{2} \mathrm{O}$ & $\mathrm{Al}+\mathrm{H}$ & $\mathrm{Al}^{3+}$ & $\begin{array}{c}\mathrm{P} \\
\mathrm{mg} / \mathrm{dm}^{3}\end{array}$ \\
\hline \multicolumn{5}{|c|}{$0,00-0,05 \mathrm{~m}$} \\
\hline Média & 6,17 & 5,75 & 0.23 & 58.85 \\
\hline Mediana & 6.17 & 5.61 & 0.20 & 57.66 \\
\hline Máximo & 7.47 & 8.42 & 0.60 & 135.4 \\
\hline Mínimo & 5.12 & 3.80 & 0.10 & 8.50 \\
\hline${ }^{1} \mathrm{DP}$ & 0.42 & 1.06 & 0.14 & 32.89 \\
\hline Variância & 0,18 & 1.13 & 0.02 & 1082.2 \\
\hline${ }^{2} \mathrm{CV} \%$ & 6.80 & 18.7 & 60.8 & 55.88 \\
\hline Assimetria & 0.01 & 0.31 & 0.88 & 0.476 \\
\hline Curtose & 0.16 & -0.35 & -0.15 & -0.707 \\
\hline${ }^{3} \mathrm{~d}$ & $0.08 *$ & $0.06^{*}$ & $0.22 *$ & $0.161 *$ \\
\hline \multicolumn{5}{|c|}{$0,05-0,10 \mathrm{~m}$} \\
\hline Média & 6.48 & 5.15 & 0.26 & 62.58 \\
\hline Mediana & 6.49 & 4.95 & 0.30 & 58.18 \\
\hline Máximo & 7.60 & 8.25 & 0.40 & 133.0 \\
\hline Mínimo & 5.35 & 2.15 & 0.10 & 12.33 \\
\hline${ }^{1} \mathrm{DP}$ & 0.47 & 1.44 & 0.06 & 33.63 \\
\hline Variância & 0.22 & 2.09 & 0.005 & 1131.1 \\
\hline${ }^{2} \mathrm{CV} \%$ & 7.25 & 27.96 & 26.53 & 53.73 \\
\hline Assimetria & -0.21 & 0.29 & -0.026 & 0.316 \\
\hline Curtose & -0.40 & -0.46 & -0.18 & -1.083 \\
\hline${ }^{3} \mathrm{~d}$ & $0.07 *$ & $0.09 *$ & $0.28 *$ & $0.04^{\mathrm{ns}}$ \\
\hline \multicolumn{5}{|c|}{$0,10-0,20 \mathrm{~m}$} \\
\hline Média & 6.30 & 4.79 & 0.35 & 50.17 \\
\hline Mediana & 6.23 & 4.79 & 0.30 & 44.75 \\
\hline Máximo & 7.39 & 8.42 & 0.50 & 112.0 \\
\hline Mínimo & 5.26 & 1.65 & 0.20 & 5.52 \\
\hline${ }^{1} \mathrm{DP}$ & 0.45 & 1.57 & 0.092 & 27.67 \\
\hline Variância & 0.20 & 2.48 & 0.009 & 765.8 \\
\hline${ }^{2} \mathrm{CV} \%$ & 7.14 & 32.78 & 26.29 & 55.15 \\
\hline Assimetria & 0.16 & 0.38 & 0.103 & 0.379 \\
\hline Curtose & -0.14 & -0.170 & -0.810 & -0.776 \\
\hline${ }^{3} \mathrm{~d}$ & $0.10^{*}$ & $0.08 *$ & $0.23 *$ & $0.73 *$ \\
\hline
\end{tabular}

DP: desvio padrão; ${ }^{2} \mathrm{CV}$ : coeficiente de variação; ${ }^{3} \mathrm{~d}$ : teste de normalidade Kolmogorov-Smirnov; *significativo a 5 \% de probabilidade.

Na tabela 2, são apresentados os parâmetros dos semivariogramas ajustados aos modelos teóricos que melhor descrevem o comportamento da variabilidade espacial dos atributos químicos do solo. Os resultados da validação cruzada (VC) foram superiores a 0,76 para todas as variáveis, o que assegura bom desempenho da interpolação pela krigagem ordinária. Esses resultados são corroborados com os coeficientes de determinação $\mathrm{R}^{2}$ acima 0,78 significando que os semivariogramas estão bem ajustados. Conforme a classificação dada por Azevedo (2004), esses resultados mostram, em geral, ajustes dos semivariogramas que possibilitam a obtenção dos mapas da distribuição espacial dos atributos de forma confiável.

Os resultados da análise geoestatística mostram que todos os atributos analisados apresentaram estrutura de dependência espacial. Para tais variáveis que apresentaram dependência espacial, ajustaram-se aos modelo exponencial e esférico, concordando com vários outros resultados que indicam este modelo como o que melhor se ajusta a esses atributos do solo (SOUZA et al., 2004). A variável alumínio na profundidade $0,10-0,20 \mathrm{~m}$ não apresentou estrutura de dependência espacial, na qual apresentou efeito pepita puro (EPP), significando que as amostras são independentes para distâncias maiores que a menor distância entre as amostras utilizadas nesse estudo.

A análise do grau de dependência espacial (GDE) proposto por Cambardella et al. (1994), que avalia em termos proporcionais o efeito pepita sobre o patamar $(\mathrm{C} 0 /(\mathrm{C} 0+\mathrm{C} 1) .100)$, em que são considerados de dependência espacial forte os semivariogramas que têm um GDE $\leq 25 \%$ do patamar; dependência espacial moderada, quando o GDE está entre 25 e $75 \%$, e dependência fraca, quando o GDE é > $75 \%$ do patamar. Verificou-se que $\mathrm{pH}$ em $\mathrm{H}_{2} \mathrm{O}$, acidez potencial e alumínio apresentaram GDE forte, com exceção da profundidade $0,05-0,10 \mathrm{~m}$ da variável alumínio que apresentou GDE moderada.

$\mathrm{O}$ alcance estabelece o limite de dependência espacial entre as amostras, isto é, para distâncias iguais ou menores que o alcance, diz-se que os valores vizinhos de uma variável estão espacialmente correlacionados e podem ser utilizados para se estimar valores em qualquer ponto entre eles (REICHARDT, 1985; TRANGMAR et al., 1985). O valor do alcance nos semivariogramas têm uma importância considerável na determinação do limite da dependência espacial, o que pode ser também um indicativo do intervalo entre unidades de mapeamento de solos (WEBSTER, 2000). 
As variáveis de $\mathrm{pH}$ em $\mathrm{H}_{2} \mathrm{O}$, acidez potencial e alcance não estão dentro dos limites estipulados pela malha alumínio estudadas apresentaram diferentes alcances de amostral de $72 \mathrm{~m}$, indicando assim a descontinuidade destes dependência espacial mostrando que alguns destes valores de atributos na área de estudo.

Tabela 2. Modelos e parâmetros estimados aos semivariogramas do pH em água, $\mathrm{H}+\mathrm{Al}$, $\mathrm{Al}$ e $\mathrm{P}$ disponível do solo em área de Terra Preta Arqueológica sob cultivo de feijão no município de Novo Aripuanã - AM.

\begin{tabular}{|c|c|c|c|c|}
\hline Parâmetros & $\mathrm{pH} \mathrm{em} \mathrm{H}_{2} \mathrm{O}$ & $\mathrm{H}+\mathrm{Al}$ & $\mathrm{Al}^{3+}$ & $\mathrm{P}$ \\
\hline Geoestatísticos & & \multicolumn{2}{|c|}{ cmolc $/ \mathrm{kg}^{-1}$} & $\mathrm{mg} / \mathrm{dm}^{3}$ \\
\hline \multicolumn{5}{|c|}{$0,00-0,05 \mathrm{~m}$} \\
\hline Modelo & ${ }^{1} \operatorname{Exp}$ & ${ }^{1} \operatorname{Exp}$ & ${ }^{1} \operatorname{Exp}$ & ${ }^{1} \operatorname{Exp}$ \\
\hline${ }^{1}\left(C_{0}\right)$ & 0.035 & 0.461 & 0.015 & 320.0 \\
\hline${ }^{2}\left(\mathrm{C}_{0}+\mathrm{C}_{1}\right)$ & 0.225 & 3.430 & 0.071 & 912.8 \\
\hline $3 \mathrm{a}(\mathrm{m})$ & 18.30 & 15.00 & 632.7 & 11.97 \\
\hline${ }^{4} R^{2}$ & 0.973 & 0.915 & 0.881 & 0.920 \\
\hline${ }^{5} \mathrm{GDE}(\%)$ & 15.55 & 13.44 & 21.12 & 35.08 \\
\hline${ }^{6} \mathrm{VC} \%$ & 0.980 & 0.862 & 0.817 & 0.789 \\
\hline Modelo & Esf & ${ }^{1} \operatorname{Exp}$ & Exp & Esf \\
\hline${ }^{1}\left(C_{0}\right)$ & 0.080 & 0.589 & 0.054 & 510.00 \\
\hline${ }^{2}\left(\mathrm{C}_{0}+\mathrm{C}_{1}\right)$ & 0.253 & 3.300 & 0.087 & 1664.0 \\
\hline $3(m)$ & 33.70 & 22.50 & 28.00 & 81.00 \\
\hline${ }^{4} \mathrm{R}^{2}$ & 0.806 & 0.895 & 0.950 & 0.902 \\
\hline${ }^{5} \mathrm{GDE}(\%)$ & 31.62 & 17.84 & 62.06 & 30.64 \\
\hline${ }^{6} \mathrm{VC} \%$ & 0.935 & 0.905 & 0.897 & 0.955 \\
\hline Modelo & Exp & Exp & EPP & ${ }^{1} \operatorname{Exp}$ \\
\hline${ }^{1}\left(C_{0}\right)$ & 0.020 & 0.443 & - & 76.00 \\
\hline${ }^{2}\left(C_{0}+C_{1}\right)$ & 0.180 & 2.760 & - & 720.0 \\
\hline $3 \mathrm{a}(\mathrm{m})$ & 10.02 & 12.90 & - & 8.73 \\
\hline${ }^{4} \mathrm{R}^{2}$ & 0.783 & 0.791 & - & 0.854 \\
\hline${ }^{5} \mathrm{GDE}(\%)$ & 11.11 & 16.05 & - & 10.55 \\
\hline${ }^{6} \mathrm{VC} \%$ & 0.784 & 0.766 & - & 0.894 \\
\hline Parâmetros & $\mathrm{pH} \mathrm{em} \mathrm{H}_{2} \mathrm{O}$ & $\mathrm{H}+\mathrm{Al}$ & $\mathrm{Al}^{3+}$ & $\mathrm{P}$ \\
\hline Geoestatísticos & & \multicolumn{2}{|c|}{ cmolc $/ \mathrm{kg}^{-1}$} & $\mathrm{mg} / \mathrm{dm}^{3}$ \\
\hline \multicolumn{5}{|c|}{$0,00-0,05 \mathrm{~m}$} \\
\hline Modelo & ${ }^{1} \operatorname{Exp}$ & ${ }^{1} \operatorname{Exp}$ & ${ }^{\mathrm{I}} \operatorname{Exp}$ & ${ }^{1} \operatorname{Exp}$ \\
\hline${ }^{\mathrm{I}}\left(\mathrm{C}_{0}\right)$ & 0.035 & 0.461 & 0.015 & 320.0 \\
\hline${ }^{2}\left(\mathrm{C}_{0}+\mathrm{C}_{1}\right)$ & 0.225 & 3.430 & 0.071 & 912.8 \\
\hline $3(\mathrm{~m})$ & 18.30 & 15.00 & 632.7 & 11.97 \\
\hline${ }^{4} \mathrm{R}^{2}$ & 0.973 & 0.915 & 0.881 & 0.920 \\
\hline${ }^{5} \mathrm{GDE}(\%)$ & 15.55 & 13.44 & 21.12 & 35.08 \\
\hline${ }^{6} \mathrm{VC} \%$ & 0.980 & 0.862 & 0.817 & 0.789 \\
\hline Modelo & Esf & ${ }^{1} \operatorname{Exp}$ & Exp & Esf \\
\hline${ }^{1}\left(C_{0}\right)$ & 0.080 & 0.589 & 0.054 & 510.00 \\
\hline${ }^{2}\left(\mathrm{C}_{0}+\mathrm{C}_{1}\right)$ & 0.253 & 3.300 & 0.087 & 1664.0 \\
\hline $3 a(m)$ & 33.70 & 22.50 & 28.00 & 81.00 \\
\hline${ }^{4} R^{2}$ & 0.806 & 0.895 & 0.950 & 0.902 \\
\hline${ }^{5} \mathrm{GDE}(\%)$ & 31.62 & 17.84 & 62.06 & 30.64 \\
\hline${ }^{6} \mathrm{VC} \%$ & 0.935 & 0.905 & 0.897 & 0.955 \\
\hline Modelo & Exp & Exp & EPP & ${ }^{1} \operatorname{Exp}$ \\
\hline${ }^{1}\left(C_{0}\right)$ & 0.020 & 0.443 & - & 76.00 \\
\hline${ }^{2}\left(\mathrm{C}_{0}+\mathrm{C}_{1}\right)$ & 0.180 & 2.760 & - & 720.0 \\
\hline $3 \mathrm{a}(\mathrm{m})$ & 10.02 & 12.90 & - & 8.73 \\
\hline${ }^{4} \mathrm{R}^{2}$ & 0.783 & 0.791 & - & 0.854 \\
\hline${ }^{5} \mathrm{GDE}(\%)$ & 11.11 & 16.05 & - & 10.55 \\
\hline${ }^{6} \mathrm{VC} \%$ & 0.784 & 0.766 & - & 0.894 \\
\hline
\end{tabular}

Esf.: Esférico; Exp.: Exponencial; ${ }^{1} \mathrm{C}_{0}$ : efeito pepita; ${ }^{2} \mathrm{C}_{0}+\mathrm{C}_{1}$ : patamar; ${ }^{3}$ a: alcance $(\mathrm{m}) ;{ }^{4} \mathrm{R}^{2}$ : coeficiente de determinação; ${ }^{5} \mathrm{GDE} \%$ : grau de dependência espacial e; ${ }^{6}$ VC: validação cruzada.

\section{CONCLUSÕES}

As características químicas estudadas apresentaram estrutura de dependência espacial, com exceção da variável alumínio que indicou efeito pepita puro.

As variáveis em estudo apresentaram diferentes valores de alcance de 8,73 a $81,00 \mathrm{~m}$, estando dentro do limite estabelecido pela malha de estudo.

\section{REFERÊNCIAS}

AZEVEDO, E. C. Uso da geoestatística e de recursos de geoprocessamento no diagnóstico da degradação de um solo argiloso sob pastagem no Estado de Mato Grosso. 2004. 132 f. Tese (Doutorado em Água e Solo) - Universidade Estadual de Campinas, Campinas, 2004. 
CAMBARDELLA, C.A.; MOORMAN, T.B.; NOVAK, J.M.; PARKIN, T.B.; KARLEN, D.L.; TURCO, R.F.; KONOPKA, A.E. Field-scale variability of soil properties in central lowa soils. Soil Sci. Sos. Am. J., v. 58, p.1501-1511, 1994.

CAMPOS, C.C.M; SANTOS, L.A.C; SILVA, D.M.P; MANTOVANELLI, B.C; SOARES, M.D.R. Caracterização física e química de terras pretas arqueológicas e de solos não antropogênicos na região de Manicoré, Amazonas. Revista Agro Ambiente, Boa Vista-RR, v. 6, n. 2, p. 102-109, 2012.

CAMPOS, M.C.C.; MARQUES JÚNIOR, J.; PEREIRA, G.T.; FREITAS, E.V.S. Dependência espacial de atributos químicos em área cultivada com cana-de-açúcar em Pereira Barreto, SP. Rev. Ciênc. Agron., Fortaleza-CE, v.38, n.4, p.350-359, 2007.

CHENG, C. H.; LEHMANN, J. Ageing of black carbon along a temperature gradient. Chemosphere, v. 75, p. 1021-1027, 2009.

CORRÊA, G.R. Caracterização pedológica de arqueoantropossolos no Brasil: sambaquis da Região dos Lagos (RJ) e Terras pretas de índio na região do baixo rio Negro/Solimões (AM). 2007, 115f. Dissertação de Mestrado, Universidade Federal de Viçosa, Viçosa, 2007.

DIGLLE, P. J.; RIBEIRO JR., P.J. Model-based geostatistics. New York: Springer, 2007.

FAO, Food and Agriculture Organization of the United Nations. Disponível em: <http://www.fao.org/>. Acesso em: Janeiro de 2011.

EMBRAPA. Centro Nacional de Pesquisa de Solos. Manual de métodos de análise de solos. 2 ed. rev. e atual. Rio de Janeiro: EMBRAPA, 1997.212p.

FARIAS, M.S.S. de. Diagnóstico da necessidade de drenagem no perímetro irrigado de São Gonçalo. Campina Grande: UFPB, 1999. 75p.

GLASER, B. Prehistorically modified soils of central Amazonia: a model for sustainable agriculture in the twentyfirst century. PhiL. Trans. R. Soc. B, v. 362, p. 187-196, 2007.

GLASER, B.; LEHMANN, J.; ZECH, W. Ameliorating physical and chemical properties of highly weathered soils in the tropics with charcoal - a review. Biol Fertil Soils, v. 35, p. 219-230, 2002.

GONÇALVES, A.C.A.; FOLEGATTI, M.V.; MATA, J.D.V. Análises exploratória e geoestatística da variabilidade de propriedades físicas de um Argissolo Vermelho. Acta Scientiarum, Maringá-PR v. 23, n. 5, p. 1149-1157, 2001.

GREGO, C. R.; VIEIRA, S. R. Variabilidade espacial de propriedades físicas do solo em uma parcela experimental. R. Bras. Ci. Solo, 29:169-177, 2005.
ISAAKS, E.H. \& SRIVASTAVA, R.M. An introduction to applied geoestatistics. New York: Oxford University Press, p. $561,1989$.

LIANG, B. et al. Black Carbon Increases Cation Exchange Capacity in Soils. Soil Sci. Soc.Am. J, v. 70, p. 1719-1730, 2006.

LIMA, H.N. et al. Pedogenesis and pre-columbian land use of "Terra Preta Anthrosols" ("Indian black earth") of Western Amazonia. Geoderma. v. 110, p. 1-17, 2002.

MERCANTE, E.; URIBE, O. M. A.; SOUZA, E. G. variabilidade espacial e temporal da resistência mecânica do solo à penetração em áreas com e sem manejo químico localizado. Cascavel-PR, R. Bras. Ci. Solo, 27:1149-1159, 2003.

MINITAB Release 14.1. Statistical Software. US/Canada, 2000.

NOGUEIRA, M. C. S. Experimentação agronômica I. Piracicaba: Universidade de São Paulo, 2007. 463p.

REICHARDT, K. Processos de transferência no sistema soloplanta-atmosfera. Campinas, Fundação Cargill, 1985. 445p.

SOUZA, Z.M.; MARQUES JÚNIOR, J.; PEREIRA, G.T.; MOREIRA, L.F. Variabilidade espacial do $\mathrm{pH}, \mathrm{Ca}, \mathrm{Mg}$ e V\% do solo em diferentes formas do relevo sob cultivo de canade-açúcar. Ciência Rural, v. 34, p. 1763-1771, 2004.

TRANGMAR, B.B.; YOST, R.S.; UEHARA, G. Application of geostatistics to spatial studies of soil properties. Adv. Agron., New York, v.38, n.1, p.45-93, 1985.

VIEIRA, S. R. Geoestatística em estudo da variabilidade espacial do solo. In: NOVAIS, R. F. de; ALVAREZ V., V. H.; SCHAEFER, C. E. G. R. Ed. Tópicos ciência do solo Campinas: Sociedade Brasileira de Ciência do Solo, 1:1-54, 2002.

VIEIRA, S. R.; HATFIELD, T. L.; NIELSEN, D. R.; BIGGAR, J. W. Geostatistical theory and application to variability of some agronomical properties. Hilgardia, Berkeley, v. 51, n. 3, 1-75, 1983.

WARRICK, A.W.; NIELSEN, D.R. Spatial variability of soil physical properties in the field. In: HILLEL, D. (Ed.). Applications of soil physics. New York: Academic Press, 1980. cap. 2, p.319-34.

WEBSTER, R. Is soil variation random? Geoderma, Amsterdam, v.97, n.1-2, p.149-163, 2000.

WERNER, V. et al. Aplicação de fertilizantes a taxa variável em agricultura de precisão variando a velocidade de deslocamento. Revista Brasileira de Engenharia Agrícola e Ambiental, v. 11, n. 6, p. 658-663, 2007. 